\title{
Neoadjuvant chemotherapy in locally advanced cervical carcinoma: which is better, intravenous or intra-arterial?
}

This article was published in the following Dove Press journal:

OncoTargets and Therapy

26 November 2014

Number of times this article has been viewed

\section{Ting Gui* \\ Keng Shen* \\ Yang Xiang \\ Lingya Pan \\ Jinghe Lang \\ Ming Wu \\ Huifang Huang \\ Dongyan Cao \\ Jiaxin Yang}

Department of Obstetrics and Gynecology, Peking Union Medical College Hospital, Peking Union Medical College, Chinese Academy of Medical Sciences, Beijing, People's Republic of China

*These authors contributed equally to the work
Correspondence: Dongyan Cao; Jiaxin Yang

Department of Obstetrics and Gynecology, Peking Union Medical

College Hospital, Peking Union Medical College, Chinese Academy of Medical Sciences, Beijing, 100730, People's Republic of China

$\mathrm{Tel} / \mathrm{Fax}+8601065296247$

Email cdy_caodongyan@I63.com; yjx_yangjiaxin@163.com
Purpose: The aim of our study is to investigate the differences in therapeutic effects and clinical significance between intravenous systematic chemotherapy and intra-arterial interventional chemotherapy in stage Ib2-IIb cervical carcinomas.

Methods: A retrospective analysis was performed on 93 cases of intravenous and 118 cases of intra-arterial neoadjuvant chemotherapy for stage Ib2-IIb cervical carcinomas treated in Peking Union Medical College Hospital from the year 2001 to 2010.

Results: After neoadjuvant chemotherapy, the overall response rate was $84.9 \%$ versus (vs) $88.2 \%$ and the operability rate was $77.4 \%$ vs $81.4 \%$, for intravenous vs intra-arterial $(P>0.05)$. There were no significant differences in toxicities, surgical duration, perioperative blood loss, and operative complications between these two groups. Postoperative pathological examination revealed a significantly lower parametrial infiltration in the intra-arterial group $(12.5 \% \mathrm{vs}$ $38.1 \%, P<0.05)$, while the positive vaginal margin, lymph node metastasis, and intravascular tumor embolus showed no significant differences. The intravenous group and the intra-arterial group had similar recurrence rate $(16.0 \%$ vs $12.3 \%)$, distant metastasis rate $(9.1 \%$ vs $8.5 \%)$, and 5 year survival rate $(79.5 \%$ vs $84.9 \%)$, without significant differences.

Conclusion: Neoadjuvant chemotherapy with cisplatin and 5-fluorouracil are safe and effective for patients with locally advanced cervical carcinomas. The intravenous and the intra-arterial approaches present with similar chemotherapy efficacy and clinical outcome. Since it is more simple and economical, the intravenous systematic approach shows greater value in clinical application.

Keywords: cervical carcinoma, neoadjuvant chemotherapy, parametrial infiltration, intravenous systemic chemotherapy, intra-arterial interventional chemotherapy

\section{Introduction}

Cervical carcinoma is the most common gynecological malignancy in developing countries, and it remains the major cause of death from cancer. Most patients present with locally advanced diseases (stage Ib2-IIb). For such cases, it is difficult to perform surgery directly due to multiple complications, or to deliver radiation because of too bulky tumors with large hypoxic tumor areas.

Neoadjuvant chemotherapy (NACT) is proposed to treat cervical cancer with huge masses or in advanced stages before surgery or radiation. ${ }^{1}$ The rationales for the use of NACT are several. Tumor size reduction may facilitate subsequent local therapy, whether radiotherapy or surgery. This reduction can transform inoperable tumors into radically resectable ones. NACT has also been suggested to increase radiosensitivity and decrease the hypoxic cell fraction. Moreover, NACT treats the micrometastatic 
disease, preventing a significant proportion of relapses. Finally, response to NACT has been identified as an important prognostic factor in several studies. ${ }^{2-6}$ A meta-analysis of NACT followed by radical hysterectomy showed an absolute improvement of $14 \%$ in survival at 5 years. ${ }^{7}$

At present, there are two approaches for NACT, intravenous systematic chemotherapy or intra-arterial interventional chemotherapy. Intravenous administration has lower requirement of techniques and expenses, but it is assumed to produce heavier systematic side effects. Intra-arterial infusion of chemotherapeutic agents in cervical cancer has been of considerable theoretical interest based on the distinct arterial supply to the tumor-bearing areas and on mathematical and pharmacokinetic models. It is believed to markedly increase the drug concentration in the pelvic cavity and enhance the ability of killing tumor cells 10-100 times, but it is much more expensive and puts more challenges on the technicians. For the time being, there is no standard method for NACT in locally advanced cervical cancer. Are there any differences in therapeutic efficacy, side effects, and clinical outcome associated with these two approaches? How do we choose in clinical practice? Considering these questions, our study retrospectively reviewed 211 patients with stage Ib2-IIb cervical carcinomas who had received NACT either intravenously or intra-arterially at Peking Union Medical College Hospital during 2001-2010, in the hope of providing some reference in future practice.

\section{Materials and methods Patient information}

Between January 2001 and December 2010, 211 patients with stage Ib2-IIb cervical carcinomas received NACT (93 intravenous and 118 intra-arterial) in the Department of Obstetrics and Gynecology at Peking Union Medical College Hospital. Patient information, including age at onset, clinical stage, NACT (approach, regimen, and courses), surgery (surgical duration, perioperative blood loss, and complications), postoperative treatment (radiotherapy and/or chemotherapy), clinical prognosis, and follow-up, was collected from clinical database. Clinical staging was performed according to the FIGO (Féderation Internationale de Gynécologie et d'Obstétrique) criteria supplemented by pelvic examination and nuclear magnetic resonance imaging. All investigations were performed according to a protocol that was evaluated and approved by the ethics committee at Peking Union Medical College Hospital, Beijing, People's Republic of China.

\section{NACT}

All enrolled patients were randomized into intravenous and intra-arterial group, and received three cycles of NACT every
3 weeks according to the scheme PF (cisplatin $70 \mathrm{mg} / \mathrm{m}^{2}$ on the first day; 5-fluorouracil $1,000 \mathrm{mg} / \mathrm{m}^{2}$ on days $\left.1-4\right)$. Intra-arterial interventional chemotherapy was performed through the right femoral artery catheterization. Each side of the uterine artery received half of the cisplatin dosage. Catheter was retained on the severer side, maintaining 24 hour infusion of 5-fluorouracil for 4 consecutive days.

\section{Evaluation of NACT efficacy}

Efficacy of NACT was assessed 14 days after the treatment in accordance with World Health Organization criteria for solid tumors. Complete response was defined as complete disappearance of all clinically detectable diseases. Partial response was recorded as $>30 \%$ reduction in total tumor size. Stable disease was defined as $<30 \%$ decrease or $<20 \%$ increase in tumor size. Progressive disease was defined as $>20 \%$ increase in size or the appearance of new lesions. Magnetic resonance imaging was used to evaluate tumor response.

\section{Treatment after NACT}

Patients with stable or progressive diseases were referred for radiotherapy, all others underwent classical radical hysterectomy, bilateral salpingo-oophorectomy, and bilateral systematic pelvic lymph node dissection. Adjuvant radio/ chemotherapy was given in the presence of high risk factor for recurrence such as positive lymph node, parametrial infiltration, or positive surgical margins.

\section{Follow-up}

Follow-up procedures included pelvic examination, vaginal margin cytology, and nuclear magnetic resonance imaging, every 3 months for 2 years, then 6 months until the fifth year. All patients had regular follow-ups and the period was considered from the day of admission to the last visit or death.

\section{Statistical analysis}

Statistical analysis was performed with SPSS 19.0 software (IBM Corporation, Armonk, NY, USA). Chi-square test was used to analyze the categorical data. A $P$-value $<0.05$ was considered statistically significant.

\section{Results}

\section{Patient information (Table I)}

There were 93 and 118 patients in the intravenous group and intra-arterial group, respectively, with a median age of 42.8 years and 40.1 years. The ratio of squamous carcinoma was $86.0 \%$ (80/93) versus (vs) 80.5\% (95/118), respectively. Regarding the clinical stage before NACT, there were 24 cases 
Table I Patient characteristics

\begin{tabular}{|c|c|c|c|c|c|c|}
\hline \multirow[t]{2}{*}{ Group } & \multirow[t]{2}{*}{$\mathbf{N}$} & \multirow[t]{2}{*}{ Age } & \multirow{2}{*}{$\begin{array}{l}\text { Squamous } \\
\text { cell carcinoma }\end{array}$} & \multicolumn{3}{|l|}{ Clinical stage } \\
\hline & & & & lb2 & Ila & Illb \\
\hline Intravenous & 93 & 42.8 & $86.0 \%(80 / 93)$ & $25.8 \%(24 / 93)$ & $44.1 \%(4 I / 93)$ & $30.1 \%(28 / 93)$ \\
\hline Intra-arterial & 118 & 40.1 & $80.5 \%(95 / 118)$ & $29.7 \%(35 / 118)$ & $36.4 \%(43 / 118)$ & $33.9 \%(40 / 118)$ \\
\hline
\end{tabular}

at stage Ib2, 41 at stage IIa, and 28 at stage IIb, in the intravenous group; there were 35 cases at stage $\mathrm{Ib} 2,43$ at stage IIa, and 40 at stage IIb, in the intra-arterial group. No significant differences were observed in age, histological type, and clinical stage between the intravenous and the intra-arterial group.

\section{Therapeutic efficacy of NACT (Table 2)}

In the intravenous group, three and 76 patients obtained complete response and partial response, respectively, with overall response rate of $84.9 \%$ (79/93); in the intra-arterial group, six and 98 patients obtained complete response and partial response, respectively, with overall response rate of $88.2 \%$ (104/118). The operability rate after NACT was $77.4 \%$ vs $81.4 \%$, for intravenous vs intra-arterial. No significant differences were found in overall response rate and operability rate between these two groups.

\section{Toxicities of NACT (Table 3)}

The most common side effects of cisplatin and 5-fluorouracil are digestive discomfort (nausea and vomiting) and bone marrow depression (neutropenia, anemia, and thrombocytopenia). Others that are not so common include impairment of renal function, acoustic nerve, and peripheral nerves, which were not observed in our study. About $67.7 \%$ and $58.5 \%$ of patients presented with digestive discomfort, and $9.7 \%$ and $8.5 \%$ patients presented with neutropenia, for intravenous vs intra-arterial. In the intravenous group, 42 patients had mild digestive discomfort, 17 patients had moderate digestive discomfort, and four patients had severe digestive discomfort. In the intra-arterial group, 40 patients had mild digestive discomfort, 24 patients had moderate digestive discomfort, and five patients had severe digestive discomfort. Nine and ten patients presented with mild bone marrow depression, mainly referring to neutropenia, in the intravenous group and intra-arterial group, respectively. There were no significant differences in the toxicities associated with NACT between the two groups. The toxicities for most patients were tolerable.

\section{Surgery, postoperative pathology, and postoperative radiotherapy after NACT (Table 4)}

No significant differences were observed in surgical duration (195 minutes vs 184 minutes), perioperative blood loss (685 mL vs $708 \mathrm{~mL})$, and complication rate (9.6\% vs $12.7 \%$ ), for intravenous vs intra-arterial. There were six cases of urinary tube fistula and three case of thrombosis in the crural intermuscular veins in the intravenous group. The complications caused by intra-arterial interventional chemotherapy included four cases of bladder injury, five cases of second debridement due to wound dehiscence, four case of urinary tube fistula, and two case of intestinal fistula.

Postoperative pathological examination revealed that $12.5 \%$ (4/32) of patients with stage IIb cervical carcinomas who received intra-arterial interventional NACT were found to have parametrial infiltration, significantly lower than the percentage in the intravenous group $(12.5 \%$ vs $38.1 \%$, $P<0.05)$. Statistical analyses did not show significant differences in the percentage of intravascular tumor embolus (16.7\% vs $20.8 \%$ ), positive vaginal margin $(22.2 \%$ vs $18.8 \%$ ), and lymph node metastasis (18.1\% vs $23.1 \%$ ), for intravenous vs intra-arterial.

Postoperative radiotherapy was given in the presence of high risk factors for recurrence such as positive lymph node, parametrial infiltration, or positive surgical margins. Our data revealed that $44.2 \%$ and $37.8 \%$ of patients received postoperative radiotherapy, for intravenous vs intra-arterial, without significant difference statistically.

\section{Clinical outcome after NACT (Table 5)}

There were eight cases and 12 cases lost in the intravenous and the intra-arterial group, respectively. The median follow-up

Table 2 Therapeutic efficacy of neoadjuvant chemotherapy

\begin{tabular}{|c|c|c|c|c|c|c|}
\hline Group & $\mathbf{N}$ & Complete response & Partial response & Stable disease & Progressive disease & Operability rate \\
\hline Intravenous & 93 & $3.2 \%(3 / 93)$ & $81.7 \%(76 / 93)$ & $8.6 \%(8 / 93)$ & $6.5 \%(6 / 93)$ & $77.4 \%(72 / 93)$ \\
\hline Intra-arterial & 118 & $5.1 \%(6 / 118)$ & $83.1 \%(98 / 1 / 8)$ & $7.6 \%$ (9/1 18) & $4.2 \%(5 / 118)$ & $81.4 \%(96 / 118)$ \\
\hline
\end{tabular}


Table 3 Toxicities of neoadjuvant chemotherapy

\begin{tabular}{|c|c|c|c|c|c|}
\hline \multirow[t]{2}{*}{ Group } & \multirow[t]{2}{*}{$\mathbf{N}$} & \multicolumn{3}{|c|}{$\begin{array}{l}\text { Digestive discomfort } \\
\text { (nausea and vomiting) }\end{array}$} & \multirow{2}{*}{$\begin{array}{l}\text { Bone marrow } \\
\text { depression (neutropenia) } \\
\text { Mild }\end{array}$} \\
\hline & & Mild & Moderate & Severe & \\
\hline Intravenous & 93 & $45.1 \%(42 / 93)$ & $18.3 \%(17 / 93)$ & $4.3 \%(4 / 93)$ & $9.7 \%(9 / 93)$ \\
\hline Intra-arterial & 118 & $33.9 \%(40 / 1 \mid 18)$ & $20.3 \%(24 / I \mid 8)$ & $4.2 \%(5 / I I 8)$ & $8.5 \%(10 / 118)$ \\
\hline
\end{tabular}

period was 66 months (range 17-117) vs 72.5 months (range 20-151) months, for intravenous vs intra-arterial. There were no significant differences in recurrence rate ( $16.5 \%$ vs $12.3 \%$ ), distant metastasis rate (9.4\% vs $8.5 \%$ ), and 5 year survival rate $(79.5 \%$ vs $84.9 \%)$, for intravenous vs intra-arterial.

\section{Discussion}

Patients with locally advanced-stage cervical carcinomas often have pathological high-risk factors for metastasis or recurrence, and treatment has often been challenged as the local control is relatively poor due to complications or too bulky tumor masses. In 1982, Frei first put forward the concept of NACT, which referred to chemotherapy prior to surgery or radiation intending to improve the operability of cancer and ultimately survival benefits. ${ }^{8} \mathrm{Li}$ et al reported that for early stage bulky cervical cancer, NACT decreased the ratio of lymphovascular space invasion, deep stromal invasion, lymph node metastasis, and the need of adjuvant radiotherapy, and finally improve 5 year progression-free survival rate and overall survival rate, as compared with primary surgery. ${ }^{9}$ Further multivariate analysis suggested that the response to NACT is an independent prognostic factor of progression-free survival and overall survival. ${ }^{9}$ In another study, Yin et al also found that NACT plus radical surgery improved the long-term disease-free survival and overall survival of patients with locally advanced stage Ib2-IIb cervical cancer compared with radical surgery alone. ${ }^{10}$ Other cisplatin-based chemotherapy regimens have also been explored in multiple clinical studies, showing a promising clinical response in a neoadjuvant setting. ${ }^{11-16}$
Although NACT followed by surgery represents a valid alternative to primary chemoradiotherapy in young and sexually active patients, on the other hand, some concerns have also been associated with the use of NACT. The toxicity of NACT has been reported by several studies. Toxic nonhematologic reactions consisted primarily of grades 1-2 nausea and vomiting, and hematologic toxicity such as neutropenia, anemia, and thrombocytopenia. ${ }^{17}$ In addition, in patients who do not respond to chemotherapeutic drugs, the administration of other curative treatment will have been delayed unnecessarily. Moreover, some chemotherapy agents could have cross-resistance with radiotherapy, inducing the development of radioresistant cellular clones. ${ }^{18}$

So far, most researchers have been focusing on the prognosis improvement induced by NACT, while studies focusing on the differences between intravenous and intraarterial NACT have been scarce. Our study, for the first time, explored the differences between these two approaches of NACT administration. In our research, we compared the two approaches in the efficacy and side effects, and no significant differences were observed in the aspects of overall response rate, operability rate, digestive discomfort, and hematological depression. Nevertheless, our data confirmed that most patients indeed benefited from NACT, consistent with the results of previous research.

As far as surgery is concerned, in our study, the surgical duration, the perioperative blood loss, and the operative complications did not show significant differences for the two approaches of NACT. Compared with previous studies, the fistula rates in our study were relatively higher. About $90 \%$ of patients who presented with urinary or intestinal fistulas had previous history of surgery, such as cesarean

Table 4 Perioperative data and postoperative pathological examination

\begin{tabular}{|c|c|c|c|c|c|c|c|c|}
\hline \multirow[t]{2}{*}{ Group } & \multirow[t]{2}{*}{$\mathbf{N}$} & \multicolumn{3}{|l|}{ Surgery } & \multicolumn{4}{|c|}{ Postoperative pathology } \\
\hline & & $\begin{array}{l}\text { Surgical duration } \\
\text { (minutes) }\end{array}$ & $\begin{array}{l}\text { Blood loss } \\
(\mathrm{mL})\end{array}$ & $\begin{array}{l}\text { Incidence of } \\
\text { complication }\end{array}$ & $\begin{array}{l}\text { Parametrial } \\
\text { infiltration }\end{array}$ & $\begin{array}{l}\text { Positive } \\
\text { vaginal margin }\end{array}$ & $\begin{array}{l}\text { Intravascular } \\
\text { tumor embolus }\end{array}$ & $\begin{array}{l}\text { Lymph node } \\
\text { metastasis }\end{array}$ \\
\hline Intravenous & 93 & 195 & 685 & $9.6 \%(9 / 83)$ & $38.1 \%(8 / 21)$ & $22.2 \%(16 / 72)$ & $16.7 \%(12 / 72)$ & $18.1 \%(13 / 72)$ \\
\hline Intra-arterial & 118 & 184 & 708 & $12.7 \%(15 / \mid 18)$ & $12.5 \%(4 / 32)$ & $18.8 \%(18 / 96)$ & $20.8 \%(19 / 96)$ & $23.1 \%(21 / 96)$ \\
\hline
\end{tabular}


Table 5 Clinical outcome after neoadjuvant chemotherapy

\begin{tabular}{llll}
\hline Group & $\begin{array}{l}\text { Recurrence } \\
\text { rate }\end{array}$ & $\begin{array}{l}\text { Distant } \\
\text { metastasis rate }\end{array}$ & $\begin{array}{l}\text { Overall } \\
\text { survival rate }\end{array}$ \\
\hline Intravenous & $16.5 \%(14 / 85)$ & $9.4 \%(8 / 85)$ & $80.0 \%(68 / 85)$ \\
Intra-arterial & $12.3 \%(13 / 106)$ & $8.5 \%(9 / 106)$ & $84.9 \%(90 / 106)$ \\
\hline
\end{tabular}

section, ovarian cystectomy, or myomectomy. During the radical hysterectomy, tissue adhesion was the main problem, bringing a lot of difficulties in recovering the normal pelvic anatomy. Considering the postoperative pathological examination of high-risk factors, these two approaches did not show significant differences in positive vaginal margin, vascular involvement, and lymph node metastasis. In spite of this, pathological examination of stage IIb tumors revealed that parametrial infiltration rate for the intra-arterial group was significantly lower than that of the intravenous group, indicating that intra-arterial intervention was superior to intravenous systematic chemotherapy in improving the infiltration of parametrial tissues.

However, regarding the clinical outcome, the incidences of recurrence, distant metastasis, and 5 year survival were not significantly different between these two groups, suggesting that these two approaches had similar effects in improving patient prognosis. We infer that, although the intra-arterial intervention could elevate regional drug concentration dramatically, different drugs may have different distribution patterns in various organs or tissues, and the intravenous delivery may produce a longer period of effective drug concentration in vivo. More research is necessary to confirm the long-term benefits of NACT by increasing the sample size or changing the regimens.

\section{Conclusion}

NACT with cisplatin and 5-fluorouracil are safe and effective for patients with locally advanced cervical carcinomas. The intravenous and the intra-arterial approaches present with similar chemotherapy efficacy and clinical outcome. Since it is more simple and economical, the intravenous systematic approach shows greater value in clinical application.

\section{Acknowledgment}

This study was supported by the National Natural Science Foundation of China (No 81172482), and National High Technology Research and Development Program of China (No 2012AA02A507).

\section{Disclosure}

The authors report no conflict of interests in this work.

\section{References}

1. Al-Mansour Z, Verschraegen C. Locally advanced cervical cancer: what is the standard of care? Curr Opin Oncol. 2010;22(5):503-512.

2. Angioli R, Plotti F, Luvero D, et al. Feasibility and safety of carboplatin plus paclitaxel as neoadjuvant chemotherapy for locally advanced cervical cancer: a pilot study. Tumour Biol. 2014;35(3):2741-2746.

3. Ye Q, Yuan HX, Chen HL. Responsiveness of neoadjuvant chemotherapy before surgery predicts favorable prognosis for cervical cancer. J Cancer Res Clin Oncol. 2013;139(11):1887-1898.

4. Sun H, Xin J, Lu Z, Wang N, Liu N, Guo Q. Potential molecular mechanisms for improved prognosis and outcome with neoadjuvant chemotherapy prior to laparoscopic radical hysterectomy for patients with cervical cancer. Cell Physiol Biochem. 2013;32(5):1528-1540.

5. Robova H, Halaska M, Pluta M, et al. The role of neoadjuvant chemotherapy and surgery in cervical cancer. Int $J$ Gynecol Cancer. 2010;20(11 Suppl 2):S42-S46.

6. Sardi JE. Comments on neoadjuvant chemotherapy in cervical cancer. Gynecol Oncol. 2008;108(2):458-459.

7. Neoadjuvant Chemotherapy for Cervical Cancer Meta-Analysis Collaboration (NACCCMA) Collaboration. Neoadjuvant chemotherapy for locally advanced cervix cancer. Cochrane Database Syst Rev. 2004;CD001774.

8. Frei E 3rd. Clinical cancer research: an embattled species. Cancer. 1982;50(10):1979-1992.

9. Li R, Lu ST, Si JG, et al. Prognostic value of responsiveness of neoadjuvant chemotherapy before surgery for patients with stage $\mathrm{IB}(2) /$ IIA(2) cervical cancer. Gynecol Oncol. 2013;128(3):524-529.

10. Yin M, Zhao F, Lou G, et al. The long-term efficacy of neoadjuvant chemotherapy followed by radical hysterectomy compared with radical surgery alone or concurrent chemoradiotherapy on locally advancedstage cervical cancer. Int J Gynecol Cancer. 2011;21(1):92-99.

11. Lorusso D, Ramondino S, Mancini M, Zanaboni F, Ditto A, Raspagliesi F. Phase II trial on cisplatin-adriamycin-paclitaxel combination as neoadjuvant chemotherapy for locally advanced cervical adenocarcinoma. Int J Gynecol Cancer. 2014;24(4):729-734.

12. Tsubamoto H, Maeda H, Kanazawa R, et al. Phase II trial on neoadjuvant intravenous and trans-uterine arterial chemotherapy for locally advanced bulky cervical adenocarcinoma. Gynecol Oncol. 2013;129(1):129-134.

13. Mousavia AS, Vahidi S, Karimi-Zarchi M, Modarress-Gilania M, Ghaemmaghamia F. Response to neoadjuvant chemotherapy with paclitaxel and cisplatin in locally advanced cervical cancer. Eur $J$ Gynaecol Oncol. 2013;34(6):527-531.

14. Shoji T, Takatori E, Saito T, et al. Neoadjuvant chemotherapy using platinum- and taxane-based regimens for bulky stage Ib2 to IIb nonsquamous cell carcinoma of the uterine cervix. Cancer Chemother Pharmacol. 2013;71(3):657-662.

15. Ferrandina G, Distefano MG, De Vincenzo R, et al. Paclitaxel, epirubicin, and cisplatin (TEP) regimen as neoadjuvant treatment in locally advanced cervical cancer: long-term results. Gynecol Oncol. 2013;128(3):518-523.

16. Manci N, Marchetti C, Di Tucci C, et al. A prospective phase II study of topotecan and cisplatin as neoadjuvant chemotherapy in locally advanced cervical cancer. Gynecol Oncol. 2011;122(2):285-290.

17. Loizzi V, Cormio G, Vicino M, Selvaggi L. Neoadjuvant chemotherapy: an alternative option of treatment for locally advanced cervical cancer. Gynecol Obstet Invest. 2008;65(2):96-103.

18. Gonzalez-Martin A, Gonzalez-Cortijl L, Carballo N, et al. The current role of neoadjuvant chemotherapy in the management of cervical carcinoma. Gynecol Oncol. 2008;110(3 Suppl 2):S36-S40. 


\section{Publish your work in this journal}

OncoTargets and Therapy is an international, peer-reviewed, open access journal focusing on the pathological basis of all cancers, potential targets for therapy and treatment protocols employed to improve the management of cancer patients. The journal also focuses on the impact of management programs and new therapeutic agents and protocols on

patient perspectives such as quality of life, adherence and satisfaction. The manuscript management system is completely online and includes a very quick and fair peer-review system, which is all easy to use. Visit http://www.dovepress.com/testimonials.php to read real quotes from published authors.

Submit your manuscript here: http://www.dovepress.com/oncotargets-and-therapy-journal 